\title{
ON THE SINGULAR LOCUS OF CERTAIN SUBVARIETIES OF SPRINGER FIBERS
}

\author{
LuCAS FRESSE
}

\begin{abstract}
Let $x \in \operatorname{End}\left(\mathbb{K}^{n}\right)$ be an endomorphism such that $x^{2}=0$ (where $\mathbb{K}$ is an algebraically closed field). The corresponding Springer fiber $\mathcal{F}_{x}$ is the algebraic variety of $x$-stable complete flags. In the present case, $\mathcal{F}_{x}$ has a suitable decomposition into a finite number of orbits under the action of the centralizer of $x$. The closures of these orbits may be singular. In this paper, we give a combinatorial description of the singular locus of the orbit closures. In particular, we deduce a description of the singular locus of the irreducible components of $\mathcal{F}_{x}$.
\end{abstract}

\section{Introduction}

Throughout this paper, we fix an algebraically closed field $\mathbb{K}$ of arbitrary characteristic. We also fix a vector space $V$ of finite dimension $n$. A chain $\left(V_{0} \subset V_{1} \subset \cdots \subset V_{n}\right)$ of subspaces of $V$ such that $\operatorname{dim} V_{i}=i$, for all $i$, is called a complete flag. By $\mathcal{F}$, we denote the set of all the complete flags. It has a natural structure of algebraic projective variety and it admits certain remarkable subvarieties. The Schubert varieties are the most classical example.

1.1. Schubert varieties and their singular loci. Choose a basis $\left(e_{1}, \ldots, e_{n}\right)$ of $V$, and let $B \subset G L(V)$ be the subgroup of linear automorphisms whose matrix in the basis is upper triangular. Then, $\mathcal{F}$ consists of a finite number of orbits for the natural action of $B$, called Schubert cells, and their closures in the Zariski topology are called Schubert varieties. The Schubert varieties are parameterized by the elements $w \in \mathbf{S}_{n}$ of the symmetric group: each Schubert cell can be written $X_{w}^{0}:=B \cdot F_{w}$, where $F_{w}:=\left(\left\langle e_{w_{1}}, \ldots, e_{w_{i}}\right\rangle_{\mathbb{K}}\right)_{i=0}^{n}$, and thus each Schubert variety is of the form $X_{w}:=\overline{B \cdot F_{w}}$. Moreover, $\operatorname{dim} X_{w}=\ell(w)$ (the Bruhat length of $w$ ), and one has $X_{w^{\prime}} \subset \overline{X_{w}}$ if and only if $w^{\prime} \leq w$, where $\leq$ stands for the Bruhat order.

The simplicity of the combinatorics allowed V. Lakshmibai and C.S. Seshadri [7] to determine the singular locus of the Schubert varieties, in the following manner. Given $w \in \mathbf{S}_{n}$, the corresponding Bruhat graph is by definition the graph whose set of vertices is $\left\{v \in \mathbf{S}_{n}: v \leq w\right\}$ and with an edge between $v$ and $v^{\prime}$ whenever one has $v^{\prime}=v(i: j)$, where $(i: j) \in \mathbf{S}_{n}$ is the transposition that switches two distinct integers $i$ and $j$. Then, the singular locus of the Schubert variety $X_{w}$ is the union of the Schubert cells $X_{v}^{0}$ corresponding to the singular vertices $v$ of the Bruhat graph of $w$, i.e., those vertices, which are incident with more than $\ell(w)$ edges. The reason is that, for each edge $\left(v, v^{\prime}\right)$ in the Bruhat graph, one gets a projective curve in $X_{w}$

Received by the editors October 26, 2011.

1991 Mathematics Subject Classification. 14M15, 14L30, 17 B08.

Key words and phrases. Flag varieties, Springer fibers, singular locus, link patterns. 
between the points $F_{v}$ and $F_{v^{\prime}}$, giving rise to a tangent vector $z_{v, v^{\prime}} \in T_{F_{v}} X_{w}$. The vectors $z_{v, v^{\prime}}$ corresponding to the various edges $\left(v, v^{\prime}\right)$ form a basis of $T_{F_{v}} X_{w}$. Thus, $\operatorname{dim} T_{F_{v}} X_{w}$ is equal to the number of edges at $v$ in the Bruhat graph.

1.2. Springer fibers. In this paper, we are rather interested in another family of varieties of complete flags. Given a nilpotent endomorphism $x \in \operatorname{End}(V)$, we let $\mathcal{F}_{x} \subset \mathcal{F}$ be the subset of $x$-stable complete flags, i.e., flags $\left(V_{0}, \ldots, V_{n}\right)$ such that $x\left(V_{i}\right) \subset V_{i-1}$ for each $i$. Then, $\mathcal{F}_{x}$ is a closed subvariety of $\mathcal{F}$ and it is called a Springer fiber. The Springer fiber $\mathcal{F}_{x}$ is an equidimensional variety (see [10]), it is usually not irreducible, and its irreducible components may be singular. Springer fibers arise in some problems in representation theory (see, for instance, $[1,6,12,13]$ ). Their elementary properties are described in [11], and the singularity of their irreducible components has been studied recently in, e.g., [3-5], [9] and references therein.

Let $Z_{x}:=\left\{g \in G L(V): g x g^{-1}=x\right\}$ be the centralizer of $x$. Thus, $Z_{x}$ naturally acts on the complete flags and leaves the Springer fiber $\mathcal{F}_{x}$ stable. However, for $x$ arbitrary, $\mathcal{F}_{x}$ may contain an infinite number of $Z_{x}$-orbits, and the inclusion relations between the $Z_{x}$-orbit closures, or of the $Z_{x}$-orbits in the components of $\mathcal{F}_{x}$, seem to be quite complicated.

1.3. Springer fibers in the case $\boldsymbol{x}^{2}=0$. Hereafter, we focus on the situation where $x$ is an endomorphism such that $x^{2}=0$. This situation is more favorable for at least two reasons:

- Here, the Springer fiber $\mathcal{F}_{x}$ consists of a finite number of $Z_{x}$-orbits, and each orbit is driven by a special flag of the form $F_{w}$ (cf. Section 1.1) corresponding to the choice of a Jordan basis $\left(e_{1}, \ldots, e_{n}\right)$.

- The orbits are suitably parameterized by a family of graphs called link patterns, and the elementary properties of the orbits (dimension, inclusion relations between closures) are known in terms of combinatorial properties of these graphs.

In Section 2, we recall from [4], [8] the description of the $Z_{x}$-orbits of $\mathcal{F}_{x}$ that we have just summed up. Relying on this, criteria for the smoothness of the components of $\mathcal{F}_{x}$ have already been established in [4]. Here, we are able to determine the singular locus of each $Z_{x}$-orbit closure of the Springer fiber $\mathcal{F}_{x}$ (in particular, of each irreducible component), by implementing a technique analogous to the one used in the case of the Schubert varieties (cf. Section 1.1). Our main result is stated in Section 3 and the proof is given in Section 4 .

\section{Description of the $Z_{x}$-orbits of $\mathcal{F}_{x}$}

As in Section 1.3, $x \in \operatorname{End}(V)$ is an endomorphism such that $x^{2}=0$. Let $k=\operatorname{rank} x$. Thus, $x$ has exactly $k$ Jordan blocks of size 2 and $n-2 k$ Jordan blocks of size 1 . In this section, our purpose is to recall the construction of the $Z_{x}$-orbits of the Springer fiber $\mathcal{F}_{x}$. This construction was given in [8] in a slightly different setting and has been adapted to the present setting in [4]. The combinatorial objects which enter the construction are presented in the next definition.

Definition 1. Let $\mathbf{S}_{n}^{2} \subset \mathbf{S}_{n}$ denote the subset of involutive permutations, that is, permutations $\sigma \in \mathbf{S}_{n}$ such that $\sigma^{2}=1$, and let $\mathbf{S}_{n}^{2}(k) \subset \mathbf{S}_{n}^{2}$ denote the subset of 
permutations that can be written as product of $k$ pairwise disjoint transpositions like $\sigma=\left(i_{1}: j_{1}\right) \cdots\left(i_{k}: j_{k}\right)$ with $i_{1}<j_{1}, \ldots, i_{k}<j_{k}$ all distinct. The graph $P_{\sigma}$ with vertices $1, \ldots, n$ and with $k$ arcs joining $\left(i_{l}, j_{l}\right)$ (for $l=1, \ldots, k$ ) is called the link pattern associated to $\sigma$. E.g., if $\sigma=(1: 4)(2: 6)(5: 7) \in S_{8}^{2}(3)$, then

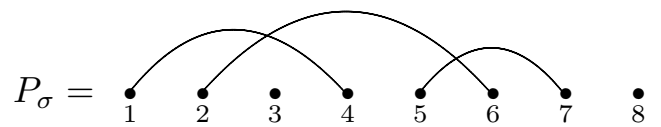

The vertices $i_{1}, \ldots, i_{k}$ (resp. $j_{1}, \ldots, j_{k}$ ) are called the left (resp. right) end points of $\sigma$ or of $P_{\sigma}$. The other vertices are called the fixed points. We will also need some auxiliary notation:

- Two arcs $\left(i_{l}, j_{l}\right),\left(i_{m}, j_{m}\right)$ are said to have a crossing if $i_{l}<i_{m}<j_{l}<j_{m}$ (i.e., the arcs intersect in $\left.P_{\sigma}\right)$; let $c(\sigma)$ denote the number of crossings of $P_{\sigma}$. A pair formed by an arc $\left(i_{l}, j_{l}\right)$ and a fixed point $p$ is said to be a bridge if $i_{l}<p<j_{l}$ (i.e., the arc spans the vertex $p$ in $P_{\sigma}$ ); let $b(\sigma)$ denote the number of bridges of $P_{\sigma}$. For example, if $\sigma$ is as above, then $b(\sigma)=c(\sigma)=2$ : the arcs $(1,4),(2,6)$, resp. $(2,6),(5,7)$, have a crossing, and the pairs $\{(1,4), 3\}$ and $\{(2,6), 3\}$ are bridges.

- Finally, given $1 \leq s<t \leq n$, we let $R_{s, t}(\sigma)$ denote the number of arcs of $P_{\sigma}$ that are contained between $s$ and $t$, i.e., the number of indices $l \in\{1, \ldots, k\}$ such that $s \leq i_{l}<j_{l} \leq t$. Furthermore, we define an order on $\mathbf{S}_{n}^{2}(k)$ by writing $\sigma^{\prime} \preceq \sigma$ if we have $R_{s, t}\left(\sigma^{\prime}\right) \leq R_{s, t}(\sigma)$ for all $s, t$.

We associate a subset of flags $\mathcal{Z}_{\sigma} \subset \mathcal{F}_{x}$ to any element $\sigma \in \mathbf{S}_{n}^{2}(k)$, as follows.

Definition 2. Let $\sigma \in \mathbf{S}_{n}^{2}(k)$. A basis $\left(e_{1}, \ldots, e_{n}\right)$ of $V$ is called a $\sigma$-basis if it satisfies the following property:

$$
x\left(e_{i}\right)= \begin{cases}e_{\sigma(i)} & \text { if } \sigma(i)<i, \\ 0 & \text { otherwise }\end{cases}
$$

A complete flag $F \in \mathcal{F}$ of the form $F=\left(\left\langle e_{1}, \ldots, e_{i}\right\rangle_{\mathbb{K}}\right)_{i=0}^{n}$, where $\left(e_{1}, \ldots, e_{n}\right)$ is a $\sigma$-basis, is called a $\sigma$-flag. We then denote by $\mathcal{Z}_{\sigma}$ the set of all the $\sigma$-flags.

It is clear that we have $\mathcal{Z}_{\sigma} \subset \mathcal{F}_{x}$. Moreover, one can see that the group $Z_{x}$ acts transitively on the set of $\sigma$-bases, thus $\mathcal{Z}_{\sigma}$ consists of a single $Z_{x}$-orbit. Furthermore, for each $F=\left(V_{0}, \ldots, V_{n}\right) \in \mathcal{Z}_{\sigma}$, one easily checks that

$$
\min \left\{j=0, \ldots, n: x\left(V_{i}\right) \subset x\left(V_{i-1}\right)+V_{j}\right\}= \begin{cases}\sigma(i) & \text { if } \sigma(i)<i, \\ 0 & \text { otherwise. }\end{cases}
$$

This implies that $\mathcal{Z}_{\sigma} \cap \mathcal{Z}_{\sigma^{\prime}} \neq \emptyset$ holds only for $\sigma=\sigma^{\prime}$. Therefore, $\sigma \mapsto \mathcal{Z}_{\sigma}$ is an injection from $\mathbf{S}_{n}^{2}(k)$ to the set of $Z_{x}$-orbits of $\mathcal{F}_{x}$. Actually this map is bijective, as mentioned among other properties in the following statement (see [4], [8]):

Proposition 1. As above, $x \in \operatorname{End}(V)$ satisfies $x^{2}=0$ and $\operatorname{rank} x=k$.

(a) The map $\sigma \mapsto \mathcal{Z}_{\sigma}$ is a bijection between $\mathbf{S}_{n}^{2}(k)$ and the set of $Z_{x}$-orbits of the Springer fiber $\mathcal{F}_{x}$.

(b) For each $\sigma \in \mathbf{S}_{n}^{2}(k)$, we have $\operatorname{dim} \mathcal{Z}_{\sigma}=\frac{(n-k)(n-k-1)}{2}+\frac{k(k-1)}{2}-b(\sigma)-c(\sigma)$. In particular, the irreducible components of $\mathcal{F}_{x}$ are the closures of the orbits 
$\mathcal{Z}_{\sigma}$ corresponding to the elements $\sigma \in \mathbf{S}_{n}^{2}(k)$ such that $P_{\sigma}$ has no crossings and no bridges.

(c) Given $\sigma, \sigma^{\prime} \in \mathbf{S}_{n}^{2}(k)$, we have $\mathcal{Z}_{\sigma^{\prime}} \subset \overline{\mathcal{Z}_{\sigma}}$ if and only if $\sigma^{\prime} \preceq \sigma$.

(d) In particular, if $\sigma_{0}=(1: n-k+1)(2: n-k+2) \cdots(k: n)$, then $\mathcal{Z}_{\sigma_{0}}$ is the only closed $Z_{x}$-orbit of $\mathcal{F}_{x}$, and we have $\operatorname{dim} \mathcal{Z}_{\sigma_{0}}=\frac{k(k-1)}{2}+\frac{(n-2 k)(n-2 k-1)}{2}$.

Example 1. Assume that $n=5, k=2$. Then, $\mathcal{F}_{x}$ consists of fifteen $Z_{x}$-orbits, corresponding to the link patterns of the following list.

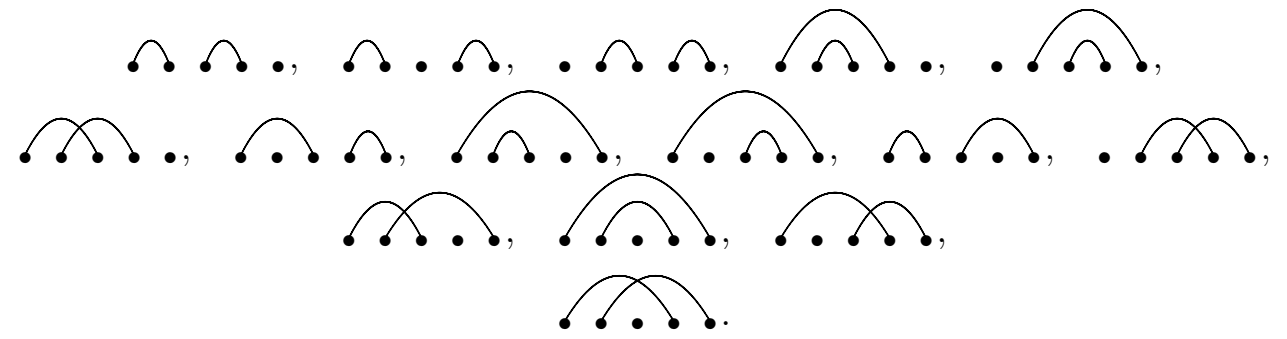

The link patterns in the first (resp. second) (resp. third) line correspond to the orbits of dimension 4 (resp. 3) (resp. 2). The only closed orbit has dimension 1 and it is represented by the link pattern in the fourth line.

Remark 1. The numbers $R_{s, t}(\sigma)$ of Definition 1 can be interpreted as follows. Given $\left(V_{0}, \ldots, V_{n}\right) \in \mathcal{Z}_{\sigma}$, by definition of $\mathcal{Z}_{\sigma}$, we have $R_{s, t}(\sigma)=\operatorname{dim}\left(V_{s-1}+x\left(V_{t}\right)\right)-s+1$ for all $1 \leq s<t \leq n$. Then, Proposition $1(\mathrm{c})$ means that the orbit closure $\overline{\mathcal{Z}_{\sigma}}$ can be described as the set of flags $F=\left(V_{0}, \ldots, V_{n}\right) \in \mathcal{F}_{x}$ such that

$$
\operatorname{dim}\left(V_{s-1}+x\left(V_{t}\right)\right) \leq s-1+R_{s, t}(\sigma) \text { for all } 1 \leq s<t \leq n .
$$

Remark 2. Fix $\left(e_{1}, \ldots, e_{n}\right)$ a basis of $V$ such that $x\left(e_{i}\right)=0$ for each $i=1, \ldots, n-k$, and $x\left(e_{n-k+i}\right)=e_{i}$ for $i=1, \ldots, k$. In this remark, we relate the orbits $\mathcal{Z}_{\sigma}$ to the special flags $F_{w}\left(w \in \mathbf{S}_{n}\right)$ of Section 1.1. Note that, by construction of $F_{w}$, we have $F_{w} \in \mathcal{F}_{x}$ if and only if $w^{-1}(i)<w^{-1}(n-k+i)$ for all $i=1, \ldots, k$. Moreover, in this case, we have $F_{w} \in \mathcal{Z}_{\sigma}$, where $\sigma \in \mathbf{S}_{n}^{2}(k)$ is the element defined by $\sigma=\prod_{i=1}^{k}\left(w^{-1}(i)\right.$ : $\left.w^{-1}(n-k+i)\right)$. It easily follows that each orbit $\mathcal{Z}_{\sigma}$ contains at least one special flag $F_{w}$, though two different special flags $F_{w}, F_{w^{\prime}}$ may belong to the same $\mathcal{Z}_{\sigma}$.

\section{Statement of the results}

In this section, we formulate our results that describe the singular locus of the $Z_{x^{-}}$ orbit closures of the Springer fiber $\mathcal{F}_{x}$. The results rely on an analogue of the Bruhat graph for link patterns, that we introduce now.

Definition 3. Given $\sigma \in \mathbf{S}_{n}^{2}(k)$, we let $X(\sigma)$ be the set of elements $\sigma^{\prime} \in \mathbf{S}_{n}^{2}(k)$ such that the link pattern $P_{\sigma^{\prime}}$ is obtained from $P_{\sigma}$ by one of the following operations:

- Either by interchanging two end points of two arcs which have a crossing (that is, $\sigma^{\prime}=\left(i: i^{\prime}\right) \sigma\left(i: i^{\prime}\right)$ where $i, i^{\prime}$ are (left or right) end points of two arcs of $P_{\sigma}$ which have a crossing);

- Or by interchanging a fixed point with the end point of an arc over it (that is, $\sigma^{\prime}=(i: p) \sigma(i: p)$ where $p$ is a fixed point of $P_{\sigma}$ and $i$ is (left or right) end point of an arc spanning $p)$. 


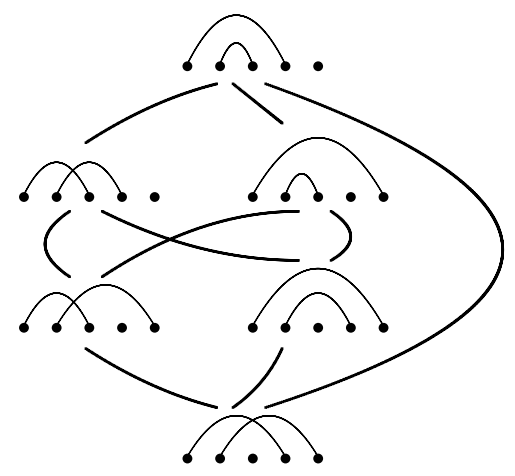

Figure 1. The graph $\mathcal{G}(\sigma)$ for $\sigma$ as in Example 2

Note that we clearly have $\sigma \prec \sigma^{\prime}$ in this case, hence $\mathcal{Z}_{\sigma} \subset \overline{\mathcal{Z}_{\sigma^{\prime}}} \backslash \mathcal{Z}_{\sigma^{\prime}}$ (cf. Proposition 1(c)). Two elements $\sigma, \sigma^{\prime} \in \mathbf{S}_{n}^{2}(k)$ are said to be adjacent if we have either $\sigma^{\prime} \in X(\sigma)$ or $\sigma \in X\left(\sigma^{\prime}\right)$. Finally, given $\sigma \in \mathbf{S}_{n}^{2}(k)$, we let $\mathcal{G}(\sigma)$ be the graph whose set of vertices is $\left\{\tau \in \mathbf{S}_{n}^{2}(k): \tau \preceq \sigma\right\}$ and with an edge between $\tau$ and $\tau^{\prime}$ whenever $\tau, \tau^{\prime}$ are adjacent.

Example 2. Let $\sigma=(1: 4)(2: 3) \in \mathbf{S}_{5}^{2}(2)$. The graph $\mathcal{G}(\sigma)$ is drawn in Figure 1 , where we represent the vertices $\tau$ by the corresponding link patterns $P_{\tau}$.

Remark 3. (a) By [8, §3], if $\tau^{\prime}$ is in the cover of $\tau$ (i.e., $\tau^{\prime} \prec \tau$ and $\tau^{\prime}$ is maximal for this property), then $\tau \in X\left(\tau^{\prime}\right)$, so that $\tau, \tau^{\prime}$ are adjacent. In particular, the graph $\mathcal{G}(\sigma)$ is always connected.

(b) Given $\tau \preceq \sigma$, the graph $\mathcal{G}(\tau)$ is the full subgraph of $\mathcal{G}(\sigma)$ whose vertices are the $\tau^{\prime} \in \mathbf{S}_{n}^{2}(k)$ such that $\tau^{\prime} \preceq \tau$.

Our main result is the following:

Theorem 1. Let $x \in \operatorname{End}(V)$ be such that $x^{2}=0$ and $\operatorname{rank} x=k$. Let $\sigma \in \mathbf{S}_{n}^{2}(k)$ and let $\mathcal{Z}_{\sigma} \subset \mathcal{F}_{x}$ be the corresponding $Z_{x}$-orbit. Let $d_{0}:=\frac{k(k-1)}{2}+\frac{(n-2 k)(n-2 k-1)}{2}$ be the dimension of the unique closed orbit of $\mathcal{F}_{x}$ (cf. Proposition 1(d)).

(a) Let $\tau \in \mathbf{S}_{n}^{2}(k)$ be such that $\tau \preceq \sigma$, so that $\mathcal{Z}_{\tau} \subset \overline{\mathcal{Z}_{\sigma}}$. Let $a(\sigma, \tau)$ denote the number of edges at the vertex $\tau$ in the graph $\mathcal{G}(\sigma)$. Then

$$
\operatorname{dim} T_{F} \overline{\mathcal{Z}_{\sigma}}=a(\sigma, \tau)+d_{0} \quad \text { for all } F \in \mathcal{Z}_{\tau} .
$$

In particular, $a(\sigma, \tau) \geq \operatorname{dim} \mathcal{Z}_{\sigma}-d_{0}$ for each vertex $\tau$.

(b) The orbit closure $\overline{\mathcal{Z}_{\sigma}}$ is smooth if and only if the graph $\mathcal{G}(\sigma)$ is regular (i.e., $a(\sigma, \tau)=\operatorname{dim} \mathcal{Z}_{\sigma}-d_{0}$ for each vertex $\left.\tau\right)$. Otherwise, the singular locus of $\overline{\mathcal{Z}_{\sigma}}$ is the union of the orbits $\mathcal{Z}_{\tau}$ which correspond to the singular vertices $\tau$ of $\mathcal{G}(\sigma)$ (i.e., those such that $\left.a(\sigma, \tau)>\operatorname{dim} \mathcal{Z}_{\sigma}-d_{0}\right)$.

In the statement, $T_{F} \overline{\mathcal{Z}_{\sigma}}$ stands for the tangent space of $\overline{\mathcal{Z}_{\sigma}}$ at the point $F$. Observe that part (b) of the theorem is an immediate consequence of part (a). Thus, our only task is to prove part (a), and this will be done in the next section.

Note that, if we apply the theorem to the element $\sigma \in \mathbf{S}_{5}^{2}(2)$ of Example 2, then we get that the orbit closure $\overline{\mathcal{Z}_{\sigma}}$ is smooth, since the graph $\mathcal{G}(\sigma)$ is regular.

The next corollary proposes a reformulation of the criterion in Theorem $1(\mathrm{~b})$. 
Corollary 1. Let $\sigma \in \mathbf{S}_{n}^{2}(k)$ and let $\tau \preceq \sigma$, so that $\mathcal{Z}_{\tau} \subset \overline{\mathcal{Z}_{\sigma}}$. Then,

$$
\operatorname{codim}_{\overline{\mathcal{Z}_{\sigma}}} \mathcal{Z}_{\tau} \leq \mid\left\{\tau^{\prime} \text { adjacent to } \tau: \tau \prec \tau^{\prime} \preceq \sigma\right\} \mid,
$$

with strict inequality if and only if $\mathcal{Z}_{\tau}$ lies in the singular locus of $\overline{\mathcal{Z}_{\sigma}}$.

Proof. In view of Remark 3(b), we have

$$
a(\sigma, \tau)=a(\tau, \tau)+\mid\left\{\tau^{\prime} \text { adjacent to } \tau: \tau \prec \tau^{\prime} \preceq \sigma\right\} \mid .
$$

Moreover, since $\mathcal{Z}_{\tau}$ lies in the regular locus of $\overline{\mathcal{Z}_{\tau}}$, Theorem 1(b) implies

$$
a(\tau, \tau)=\operatorname{dim} \mathcal{Z}_{\tau}-d_{0} .
$$

This yields

$$
\mid\left\{\tau^{\prime} \text { adjacent to } \tau: \tau \prec \tau^{\prime} \preceq \sigma\right\} \mid-\operatorname{codim}_{\overline{\mathcal{Z}_{\sigma}}} \mathcal{Z}_{\tau}=a(\sigma, \tau)-\operatorname{dim} \mathcal{Z}_{\sigma}+d_{0} .
$$

Thereby, the relations in Theorem 1(b) imply the desired conclusion.

This reformulation allows us to show that the orbit closures $\overline{\mathcal{Z}_{\sigma}}$ are regular in codimension one:

Corollary 2. Let $\sigma \in \mathbf{S}_{n}^{2}(k)$ and let $\operatorname{Sing}\left(\overline{\mathcal{Z}_{\sigma}}\right)$ be the singular locus of the orbit closure $\overline{\mathcal{Z}_{\sigma}}$. Then, $\operatorname{codim} \overline{\mathcal{Z}_{\sigma}} \operatorname{Sing}\left(\overline{\mathcal{Z}_{\sigma}}\right) \geq 2$.

Proof. We have to check that each 1-codimensional orbit $\mathcal{Z}_{\tau} \subset \overline{\mathcal{Z}_{\sigma}}$ lies outside of the singular locus of $\overline{\mathcal{Z}_{\sigma}}$. Such $\mathcal{Z}_{\tau}$ lies in the cover of $\overline{\mathcal{Z}_{\sigma}}$, thus (by Proposition $1(\mathrm{c})$ ) there is no $\tau^{\prime} \neq \sigma$ such that $\tau \prec \tau^{\prime} \preceq \sigma$. This implies that

$$
\mid\left\{\tau^{\prime} \text { adjacent to } \tau: \tau \prec \tau^{\prime} \preceq \sigma\right\} \mid \leq 1=\operatorname{codim}_{\overline{\mathcal{Z}_{\sigma}}} \mathcal{Z}_{\tau},
$$

which, by Corollary 1 , ensures that $\mathcal{Z}_{\tau}$ does not lie in the singular locus of $\overline{\mathcal{Z}_{\sigma}}$.

In the case where $\overline{\mathcal{Z}_{\sigma}}$ has maximal dimension (i.e., is a component of $\mathcal{F}_{x}$ ), N. Perrin and E. Smirnov [9] have proved that $\overline{\mathcal{Z}_{\sigma}}$ is a normal variety, which already implies that it is regular in codimension one in this case. In view of Corollary 2, we can speculate that $\overline{\mathcal{Z}_{\sigma}}$ is normal even if it is not of maximal dimension.

One can deduce from Corollary 1 a simpler criterion of singularity for the closures of the $Z_{x}$-orbits of $\mathcal{F}_{x}$, in the following manner. Let $\sigma_{0}$ be as in Proposition $1(\mathrm{~d})$, so that $\mathcal{Z}_{\sigma_{0}}$ is the only closed $Z_{x}$-orbit of $\mathcal{F}_{x}$. Then, the singular locus of $\overline{\mathcal{Z}_{\sigma}}$ is nonempty only when it contains $\mathcal{Z}_{\sigma_{0}}$. Thus, Corollary 1 implies:

Corollary 3. Let $\sigma \in \mathbf{S}_{n}^{2}(k)$. Then,

$$
\operatorname{dim} \mathcal{Z}_{\sigma}-d_{0} \leq \mid\left\{\tau \text { adjacent to } \sigma_{0}: \tau \preceq \sigma\right\} \mid
$$

with strict inequality if and only if $\overline{\mathcal{Z}_{\sigma}}$ is singular.

In the case, where $\overline{\mathcal{Z}_{\sigma}}$ has maximal dimension, Corollary 3 retrieves the conclusion of Theorem 3.1 of [2].

The next examples illustrate our results in the particular situation where $k \leq 2$.

Example 3. (a) Let $n \geq 2$ and suppose that $k=1$. Then, every $Z_{x}$-orbit closure in $\mathcal{F}_{x}$ is smooth. (b) Let $n \geq 4$ and suppose that $k=2$. Let $\sigma=(a: b)(c: d) \in \mathbf{S}_{n}^{2}(2)$ 
with $a<b, c<d$ and, say, $a<c$. Then, the orbit closure $\overline{\mathcal{Z}_{\sigma}}$ is singular exactly in the following three cases:

(i) $a<b<c<d$ and $(b>2$ and $c<n-1)$;

(ii) $a<c<b<d$ and $(a>1$ and $d<n)$;

(iii) $a<c<d<b$ and $((a>1$ and $c-a>1)$ or $(b<n$ and $b-d>1))$.

Claim (a) can be shown by applying Corollary 3, or directly as follows. Take $\sigma=(a: b) \in \mathbf{S}_{n}^{2}(1)$ (with $\left.a<b\right)$. By Proposition $1\left(\mathrm{c}\right.$ ), we can see that $\overline{\mathcal{Z}_{\sigma}}$ is the union of the orbits $\mathcal{Z}_{(i: j)}$ for $i \leq a<b \leq j$, hence $\overline{\mathcal{Z}_{\sigma}}$ is the set of flags $\left(V_{0}, \ldots, V_{n}\right)$ such that $\operatorname{Im} x \subset V_{a}, V_{b-1} \subset \operatorname{ker} x$. Then, it is straightforward to check that $\overline{\mathcal{Z}_{\sigma}}$ is an iterated bundle of base type $\left(\operatorname{Grass}_{b-2}\left(\mathbb{K}^{n-2}\right), \operatorname{Grass}_{a-1}\left(\mathbb{K}^{b-2}\right), \mathcal{F}^{(a)}, \mathcal{F}^{(b-a-1)}, \mathcal{F}^{(n-b+1)}\right)$, which implies that it is smooth (here, $\operatorname{Grass}_{l}\left(\mathbb{K}^{m}\right)$ is the variety of $l$-dimensional subspaces of $\mathbb{K}^{m}$, and $\mathcal{F}^{(m)}$ denotes the variety of complete flags of $\mathbb{K}^{m}$ ).

To show Claim (b), we proceed as follows. First, by Proposition 1(b), we find

$$
\operatorname{dim} \mathcal{Z}_{\sigma}-d_{0}= \begin{cases}2 n+a+c-b-d-5 & \text { if } a<b<c<d, \\ 2 n+a+c-b-d-4 & \text { if } a<c<b<d \\ 2 n+a+c-b-d-3 & \text { if } a<c<d<b\end{cases}
$$

Next, we enumerate the set $X\left(\sigma_{0}\right)$ of elements $\tau \in \mathbf{S}_{n}^{2}(2)$ that are adjacent to $\sigma_{0}$ : we can see that $X\left(\sigma_{0}\right)=\{(1: 2)(n-1: n),(1: n)(2: n-1),(i: n-1)(2: n)$, $(1: j)(2: n),(1: n-1)(l: n),(1: n-1)(2: m): i, j, l, m=3, \ldots, n-2\}$. A straightforward calculation allows us to determine the elements $\tau \in X\left(\sigma_{0}\right)$, which are $\preceq \sigma$ (according to the different possible configurations of $a, b, c, d$ ), and then we can apply the criterion in Corollary 3 in order to reach the conclusion of Claim (b).

Example 4. Let $n \geq 6$ and, as in Example 3(b), suppose $k=2$. Let $\sigma \in \mathbf{S}_{n}^{2}(2)$ be such that $\overline{\mathcal{Z}_{\sigma}} \subset \mathcal{F}_{x}$ has maximal dimension and is singular. Thus, $\sigma=(a: a+1)(b: b+1)$ with $a+1<b, 1<a, b<n-1$. Then (as justified below), the singular locus of $\overline{\mathcal{Z}_{\sigma}}$ is the closure of the $Z_{x}$-orbit $\mathcal{Z}_{(a-1: b+2)(a: b+1)}$. In particular, $\operatorname{Sing}\left(\overline{\mathcal{Z}_{\sigma}}\right)$ is irreducible, of codimension $2(b-a)$, and, by Example 3(b), it is smooth.

Let $\tau=(a-1: b+2)(a: b+1)$. The claimed equality $\operatorname{Sing}\left(\overline{\mathcal{Z}_{\sigma}}\right)=\overline{\mathcal{Z}_{\tau}}$ is checked in two steps:

(1) We show that the orbit $\mathcal{Z}_{\tau}$ lies in the singular locus. To do this we describe the set $X(\tau)$ of elements $\tau^{\prime} \succ \tau$ adjacent to $\tau: X(\tau)=\{(i: b+2)(a: b+1)$, $(a-1: j)(a: b+1),(a-1: b+2)(l: b+1),(a-1: b+2)(a: m):$ $i, j, l, m=a+1, \ldots, b\}$. We can see that $\tau^{\prime} \preceq \sigma$ for each $\tau^{\prime} \in X(\tau)$. Thus, $\mid\left\{\tau^{\prime}\right.$ adjacent to $\left.\tau: \tau \prec \tau^{\prime} \preceq \sigma\right\} \mid=4(b-a)>2(b-a)=\operatorname{codim}_{\overline{\mathcal{Z}_{\sigma}}} \mathcal{Z}_{\tau}$. Then, Corollary 1 implies that $\mathcal{Z}_{\tau} \subset \operatorname{Sing}\left(\overline{\mathcal{Z}_{\sigma}}\right)$. Thereby, $\overline{\mathcal{Z}_{\tau}} \subset \operatorname{Sing}\left(\overline{\mathcal{Z}_{\sigma}}\right)$.

(2) We show that, if $\tau^{\prime} \in \mathbf{S}_{n}^{2}(2)$ satisfies $\tau^{\prime} \preceq \sigma$ and $\tau^{\prime} \npreceq \tau$ (that is, $\mathcal{Z}_{\tau^{\prime}} \subset \overline{\mathcal{Z}_{\sigma}} \backslash \overline{\mathcal{Z}_{\tau}}$ ), then $\mathcal{Z}_{\tau^{\prime}}$ lies outside of $\operatorname{Sing}\left(\overline{\mathcal{Z}_{\sigma}}\right)$. Such $\tau^{\prime}$ takes the form $\tau^{\prime}=\left(i_{1}: j_{1}\right)\left(i_{2}: j_{2}\right)$ with $i_{1} \leq a<j_{1}$ and $\left(a<i_{2} \leq b<j_{2}\right.$ or $i_{2} \leq a<\min \left(j_{1}, j_{2}\right) \leq b<$ $\left.\max \left(j_{1}, j_{2}\right)\right)$. For each $\tau^{\prime}$ of this type, we consider the set $X\left(\tau^{\prime}\right)$ of elements $\tau^{\prime \prime} \succ \tau^{\prime}$ adjacent to $\tau^{\prime}$. For every configuration of $i_{1}, j_{1}, i_{2}, j_{2}$, we can check that $\left|\left\{\tau^{\prime \prime} \in X\left(\tau^{\prime}\right): \tau^{\prime \prime} \preceq \sigma\right\}\right|=\operatorname{codim}_{\mathcal{Z}_{\sigma}} \mathcal{Z}_{\tau^{\prime}}$; thus Corollary 1 implies that $\mathcal{Z}_{\tau^{\prime}} \not \subset \operatorname{Sing}\left(\overline{\mathcal{Z}_{\sigma}}\right)$. 


\section{Proof of Theorem 1}

The purpose of this section is to prove Theorem 1(a). The proof that we propose generalizes the ideas of the proof of the main theorem in [2]. We present our strategy in the next subsection.

4.1. Outline of the proof. Let $\sigma, \tau \in \mathbf{S}_{n}^{2}(k)$ such that $\tau \preceq \sigma$, and let $F \in \mathcal{Z}_{\tau}$. Thus, $F$ is a $\tau$-flag, and there is a $\tau$-basis $\left(e_{1}, \ldots, e_{n}\right)$ such that $F=\left(\left\langle e_{1}, \ldots, e_{i}\right\rangle\right)_{i=0}^{n}$. Our goal is then to compute the dimension of the tangent space $T_{F} \overline{\mathcal{Z}_{\sigma}}$.

Let $B=\left\{g \in G L(V): g e_{i} \in\left\langle e_{j}: j=i, \ldots, n\right\rangle_{\mathbb{K}}\right\}$ be the group of automorphisms that are lower triangular in the basis, and let $\Omega=B \cdot F \subset \mathcal{F}$. Thus, $\Omega$ is an open neighborhood of $F$ in $\mathcal{F}$. Moreover, for $F^{\prime} \in \Omega$, there are unique scalars $\left(\varphi_{i, j}\left(F^{\prime}\right)\right)_{1 \leq i<j \leq n}$ such that $F^{\prime}=\left(\left\langle f_{1}, \ldots, f_{i}\right\rangle_{\mathbb{K}}\right)_{i=0}^{n}$ with $f_{i}=e_{i}+\sum_{j>i} \varphi_{i, j}\left(F^{\prime}\right) e_{j}$. The maps $\varphi_{i, j}: \Omega \rightarrow \mathbb{K}$ induce an isomorphism of algebraic varieties $\Omega \cong \mathbb{K}^{\frac{n(n-1)}{2}}$. This equips $\Omega$ with a structure of vector space with $\left(\varphi_{i, j}\right)_{1 \leq i<j \leq n}$ as basis of its dual, and such that $F$ identifies with the zero vector. Let $\left(\varepsilon_{i, j}\right)_{1<i<j<n} \subset \Omega$ be the dual basis. The tangent space $\mathcal{T}:=T_{F} \overline{\mathcal{Z}_{\sigma}}$ identifies to a vector subspace of $\Omega$. Let $\mathcal{T}^{\perp}=\left\{\varphi \in \Omega^{*}: \varphi(\varepsilon)=0 \quad \forall \varepsilon \in \mathcal{T}\right\}$.

In what follows, we aim to construct a family of $d_{0}+a(\sigma, \tau)$ linearly independent vectors of $\mathcal{T}$, and a family of $\frac{n(n-1)}{2}-d_{0}-a(\sigma, \tau)$ linearly independent elements of $\mathcal{T}^{\perp}$. In view of the relation $\operatorname{dim} \mathcal{T}+\operatorname{dim} \mathcal{T}^{\perp}=\operatorname{dim} \Omega=\frac{n(n-1)}{2}$, this will imply the equality $\operatorname{dim} \mathcal{T}=d_{0}+a(\sigma, \tau)$, and the proof will be complete.

4.2. Auxiliary statement. In this subsection, we formulate an explicit statement which describes the construction of the linearly independent elements of $\mathcal{T}$ and $\mathcal{T}^{\perp}$ outlined above, and which will imply Theorem $1(\mathrm{a})$.

We first need some notation. Given $1 \leq i<j \leq n$, we let $\omega_{i, j}(\tau) \in \mathbf{S}_{n}^{2}(k)$ be the element whose link pattern $P_{\omega_{i, j}(\tau)}$ is obtained from $P_{\tau}$ by interchanging the vertices $i$ and $j$ (i.e., $\left.\omega_{i, j}(\tau)=(i: j) \tau(i: j)\right)$. Let $I$ be the set of pairs $(i, j)$ with $1 \leq i<j \leq n$. We introduce four subsets $I_{0}, I_{1}^{-}, I_{1}^{+}, I_{2}$.

- Let $I_{0}$ be the set of pairs $(i, j) \in I$ such that $i, j$ are both fixed points of $P_{\tau}$ (i.e., $\tau_{i}=i$ and $\tau_{j}=j$ ) or both right end points (i.e., $\tau_{i}<i$ and $\tau_{j}<j$ ).

- Let $I_{1}^{-}$be the set of pairs $(i, j) \in I$ satisfying one of the following conditions:

(a) $i$ is a fixed point and $j$ is the left end point of an arc in the link pattern $P_{\tau}$, that is, $\tau_{i}=i<j<\tau_{j}$;

(b) $i$ is right end point and $j$ is fixed point, that is, $\tau_{i}<i<j=\tau_{j}$;

(c) $i$ is right end point and $j$ is left end point, that is, $\tau_{i}<i<j<\tau_{j}$;

(d) $i$ and $j$ are left end points and the arc starting at $i$ is over the arc starting at $j$, that is, $i<j<\tau_{j}<\tau_{i}$.

- Let $I_{1}^{+}$be the set of pairs $(i, j) \in I$ that satisfy one of the conditions:

(a) $i$ is a fixed point and $j$ is the right end point of an arc over $i$, that is, $\tau_{j}<\tau_{i}=i<j$

(b) $j$ is fixed point and $i$ is left end point of an arc over $j$, that is, $i<j=$ $\tau_{j}<\tau_{i}$

(c) $i$ and $j$ are the left end points of two arcs that have a crossing, that is, $i<j<\tau_{i}<\tau_{j}$ 
(d) $i$ and $j$ are respectively left- and right end points of two arcs that have a crossing and $j$ is under the arc starting at $i$, that is, $\tau_{j}<i<j<\tau_{i}$.

- Let $I_{2}=I \backslash\left(I_{0} \cup I_{1}^{-} \cup I_{1}^{+}\right)$.

Note that $\left|I_{0}\right|=d_{0}$. A careful comparison with Definition 3 shows that the maps $I_{1}^{-} \rightarrow$ $\left\{\tau^{\prime}\right.$ adjacent to $\left.\tau: \tau^{\prime} \prec \tau\right\},(i, j) \mapsto \omega_{i, j}(\tau)$ and $I_{1}^{+} \rightarrow\left\{\tau^{\prime}\right.$ adjacent to $\left.\tau: \tau^{\prime} \succ \tau\right\}$, $(i, j) \mapsto \omega_{i, j}(\tau)$ are bijective.

Proposition 2. Let $(i, j) \in I$.

(i) If $(i, j) \in I_{0}$, then $\varepsilon_{i, j} \in \mathcal{T}$, or $\varepsilon_{i, j}+\varepsilon_{i^{\prime}, j^{\prime}} \in \mathcal{T}$ for some $i^{\prime}<i, j^{\prime}<j$.

(ii) If $(i, j) \in I_{1}^{-} \cup I_{1}^{+}$and $\omega_{i, j}(\tau) \preceq \sigma$, then $\varepsilon_{i, j} \in \mathcal{T}$.

(iii) If $(i, j) \in I_{1}^{-} \cup I_{1}^{+}$and $\omega_{i, j}(\tau) \npreceq \sigma$, then $\varphi_{i, j} \in \mathcal{T}^{\perp}$, or $\varphi_{i, j}-\varphi_{i^{\prime}, j^{\prime}} \in \mathcal{T}^{\perp}$ for some $i^{\prime}>i, j^{\prime}>j$.

(iv) If $(i, j) \in I_{2}$, then $\varphi_{i, j} \in \mathcal{T}^{\perp}$, or $\varphi_{i, j}-\varphi_{i^{\prime}, j^{\prime}} \in \mathcal{T}^{\perp}$ for some $i^{\prime}>i, j^{\prime}>j$.

Observe that $\left|\left\{(i, j) \in I_{1}^{-} \cup I_{1}^{+}: \omega_{i, j}(\tau) \preceq \sigma\right\}\right|=a(\sigma, \tau)$. Thus, parts (i) and (ii) of the proposition provide $d_{0}+a(\sigma, \tau)$ linearly independent elements of $\mathcal{T}$. Parts (iii) and (iv) provide $\frac{n(n-1)}{2}-d_{0}-a(\sigma, \tau)$ linearly independent elements of $\mathcal{T}^{\perp}$. Thereby, in view of what is explained in Section 4.1, it suffices to show Proposition 2 in order to get Theorem 1(a). The proof of the proposition is cut into the following subsections.

4.3. Proof of Proposition 2(i). Let $(i, j) \in I_{0}$. Thus, we have either $\left(i=\tau_{i}\right.$ and $\left.j=\tau_{j}\right)$ or $\left(\tau_{i}<i\right.$ and $\left.\tau_{j}<j\right)$. For each $t \in \mathbb{K}$, we define an element $g_{t} \in Z_{x}$ by letting $g_{t}\left(e_{i}\right)=e_{i}+t e_{j}, g_{t}\left(e_{\tau_{i}}\right)=e_{\tau_{i}}+t e_{\tau_{j}}$, and $g_{t}\left(e_{l}\right)=e_{l}$ for each $l \in\{1, \ldots, n\} \backslash\left\{i, \tau_{i}\right\}$. The curve $\left\{g_{t}(F): t \in \mathbb{K}\right\}$ lies in $\mathcal{Z}_{\tau}$, hence in $\overline{\mathcal{Z}_{\sigma}}$, and the tangent vector at $t=0$ to this curve is thereby an element of $\mathcal{T}$.

If $\left(\tau_{i}<i, \tau_{j}<j\right.$ and $\left.\tau_{i}<\tau_{j}\right)$, then we have $g_{t}(F)=\left(\left\langle f_{1}, \ldots, f_{l}\right\rangle_{\mathbb{K}}\right)_{l=0}^{n}$ with $f_{i}=e_{i}+t e_{j}, f_{\tau_{i}}=e_{\tau_{i}}+t e_{\tau_{j}}$, and $f_{l}=e_{l}$ for each $l \notin\left\{i, \tau_{i}\right\}$. In other words, $g_{t}(F)=t\left(\varepsilon_{i, j}+\varepsilon_{\tau_{i}, \tau_{j}}\right)$. Thus, the tangent vector at $t=0$ to the curve $\left\{g_{t}(F): t \in \mathbb{K}\right\}$ is $\varepsilon_{i, j}+\varepsilon_{\tau_{i}, \tau_{j}}$. This implies that $\varepsilon_{i, j}+\varepsilon_{\tau_{i}, \tau_{j}} \in \mathcal{T}$ in this case.

If $\left(i=\tau_{i}\right.$ and $\left.j=\tau_{j}\right)$ or $\left(\tau_{i}<i, \tau_{j}<j\right.$ and $\left.\tau_{i}>\tau_{j}\right)$, then we have $g_{t}(F)=$ $\left(\left\langle f_{1}, \ldots, f_{l}\right\rangle_{\mathbb{K}}\right)_{l=0}^{n}$ with $f_{i}=e_{i}+t e_{j}$ and $f_{l}=e_{l}$ for each $l \neq i$. Thereby, $g_{t}(F)=t \varepsilon_{i, j}$. We therefore obtain that $\varepsilon_{i, j} \in \mathcal{T}$ in this case. This shows Proposition 2(i).

4.4. Proof of Proposition 2(ii). We first concentrate on the subset $I_{1}^{-}$. Since $\tau \preceq \sigma$, each $(i, j) \in I_{1}^{-}$satisfies $\omega_{i, j}(\tau) \prec \sigma$. Thus, we have to show that $\varepsilon_{i, j} \in \mathcal{T}$ for each $(i, j) \in I_{1}^{-}$.

Recall the conditions (a) to (d) of the definition of $I_{1}^{-}$. If $(i, j)$ satisfies one of the conditions (a) to (c), then the automorphism $g_{t} \in G L(V)$ defined by $g_{t}\left(e_{l}\right)=e_{l}$ for $l \neq i$ and $g_{t}\left(e_{i}\right)=e_{i}+t e_{j}$ satisfies $g_{t} \in Z_{x}$. If $(i, j)$ satisfies condition (d), then we rather consider the map $g_{t} \in G L(V)$ given by $g_{t}\left(e_{l}\right)=e_{l}$ for all $l \notin\left\{i, \tau_{i}\right\}$, $g_{t}\left(e_{i}\right)=e_{i}+t e_{j}$, and $g_{t}\left(e_{\tau_{i}}\right)=e_{\tau_{i}}+t e_{\tau_{j}}$; again, we have $g_{t} \in Z_{x}$. Thus, in both cases, the curve $\left\{g_{t}(F): t \in \mathbb{K}\right\}$ lies in $\overline{\mathcal{Z}_{\sigma}}$, and the tangent vector at $t=0$ to this curve is an element of $\mathcal{T}$. In both cases, we can see that $g_{t}(F)=\left(\left\langle f_{1}, \ldots, f_{l}\right\rangle_{\mathbb{K}}\right)_{l=0}^{n}$ with $f_{i}=e_{i}+t e_{j}$ and $f_{l}=e_{l}$ for each $l \neq i$. So, $g_{t}(F)=t \varepsilon_{i, j}$. Differentiating at $t=0$, we obtain that $\varepsilon_{i, j} \in \mathcal{T}$, as claimed.

Next, let us focus on the subset $I_{1}^{+}$: we take $(i, j) \in I_{1}^{+}$satisfying $\omega_{i, j}(\tau) \preceq \sigma$, and we have to show that $\varepsilon_{i, j} \in \mathcal{T}$. 
Set $e_{i}^{\prime}=e_{j}, e_{j}^{\prime}=e_{i}$, and $e_{l}^{\prime}=e_{l}$ for $l \notin\{i, j\}$. Using that $(i, j) \in I_{1}^{+}$, we can check that $\left(e_{1}^{\prime}, \ldots, e_{n}^{\prime}\right)$ is a $\omega_{i, j}(\tau)$-basis. So $F^{\prime}:=\left(\left\langle e_{1}^{\prime}, \ldots, e_{l}^{\prime}\right\rangle_{\mathbb{K}}\right)_{l=0}^{n}$ is a $\omega_{i, j}(\tau)$-flag, that is, $F^{\prime} \in \mathcal{Z}_{\omega_{i, j}(\tau)}$. Since $\omega_{i, j}(\tau) \preceq \sigma$, by Proposition 1 , we deduce that $F^{\prime} \in \overline{\mathcal{Z}_{\sigma}}$.

Recall the conditions (a) to (d) of the definition of $I_{1}^{+}$. If $(i, j)$ satisfies (a), (b) or (d), then we consider $g_{t} \in G L(V)$ defined by $g_{t}\left(e_{j}\right)=e_{j}+t e_{i}$, and $g_{t}\left(e_{l}\right)=e_{l}$ for all $l \neq j$. If $(i, j)$ satisfies $(\mathrm{c})$, then we rather take $g_{t} \in G L(V)$ such that $g_{t}\left(e_{j}\right)=e_{j}+t e_{i}$, $g_{t}\left(e_{\tau_{j}}\right)=e_{\tau_{j}}+t e_{\tau_{i}}$, and $g_{t}\left(e_{l}\right)=e_{l}$ for all $l \notin\left\{j, \tau_{j}\right\}$. In both cases, we see that $g_{t} \in Z_{x}$, hence the curve $\left\{g_{t}\left(F^{\prime}\right): t \in \mathbb{K}\right\}$ is contained in $\overline{\mathcal{Z}_{\sigma}}$. In both cases, we can see that $F=\lim _{t \rightarrow \infty} g_{t}\left(F^{\prime}\right)$. In fact, for each $t \in \mathbb{K}^{\times}$, we have $g_{t}\left(F^{\prime}\right)=\left(\left\langle f_{1}, \ldots, f_{l}\right\rangle_{\mathbb{K}}\right)_{l=0}^{n}$ where $f_{l}=e_{l}$ for $l \neq i$ and $f_{i}=e_{i}+t^{-1} e_{j}$. In other words, $g_{t}\left(F^{\prime}\right)=t^{-1} \varepsilon_{i, j}$. In particular, this yields $t \varepsilon_{i, j} \in \overline{\mathcal{Z}_{\sigma}}$ for all $t \in \mathbb{K}$. Differentiating at $t=0$, we obtain $\varepsilon_{i, j} \in \mathcal{T}$. Proposition 2(ii) ensues.

4.5. Proof of Proposition 2(iii). Let $(i, j) \in I_{1}^{-} \cup I_{1}^{+}$be such that $\omega_{i, j}(\tau) \npreceq \sigma$. We have necessarily $(i, j) \in I_{1}^{+}$(because each $(l, m) \in I_{1}^{-}$satisfies $\omega_{l, m}(\tau) \prec \tau$ and so $\left.\omega_{l, m}(\tau) \preceq \sigma\right)$, hence $(i, j)$ satisfies one of the conditions (a) to (d) in the definition of $I_{1}^{+}$formulated in Section 4.2.

Recall that $R_{s, t}(\tau)$ (for $1 \leq s<t \leq n$ ) denotes the number of arcs between $s$ and $t$ in the link pattern $P_{\tau}$. On one hand, since $\tau$ is such that $\tau \preceq \sigma$, one has $R_{s, t}(\tau) \leq$ $R_{s, t}(\sigma)$ for each $s, t$, and moreover, in view of the definition of $\omega_{i, j}(\tau)$ and of the set $I_{1}^{+}$, one has $R_{s, t}(\tau) \leq R_{s, t}\left(\omega_{i, j}(\tau)\right) \leq R_{s, t}(\tau)+1$. On the other hand, the assumption that $\omega_{i, j}(\tau) \npreceq \sigma$ implies that there are $s<t$ such that $R_{s, t}\left(\omega_{i, j}(\tau)\right)>R_{s, t}(\sigma)$. We fix a pair $(s, t)$ with the latter property. For this pair $(s, t)$, we therefore have

$$
R_{s, t}(\sigma)=R_{s, t}(\tau) \quad \text { and } \quad R_{s, t}\left(\omega_{i, j}(\tau)\right)=R_{s, t}(\tau)+1 .
$$

We abbreviate $r=R_{s, t}(\sigma)$. By the equality $R_{s, t}(\tau)=R_{s, t}(\sigma)$, there are indices $i_{1}<\cdots<i_{r}$ such that $s \leq \tau_{i_{p}}<i_{p} \leq t$ for each $p$. By Remark 1, every element $F^{\prime}=\left(V_{0}, \ldots, V_{n}\right) \in \Omega \cap \overline{\mathcal{Z}_{\sigma}}$ satisfies

$$
\operatorname{dim}\left(V_{s-1}+x\left(V_{t}\right)\right) \leq s+r-1 .
$$

In what follows, we will rely on relation $(*)$ and on the indices $i_{1}, \ldots, i_{r}$ in order to deduce certain relations in the tangent space $\mathcal{T}$.

In the next step, we determine all the possible configurations of $s, t, i, \tau_{i}, j, \tau_{j}$.

- Assume first that $(i, j)$ satisfies condition (a) of the definition of $I_{1}^{+}$, i.e., $\tau_{j}<\tau_{i}=i<j$. In this case, $\tau, \omega_{i, j}(\tau)$ only differ by the fact that the link pattern $P_{\tau}$ contains $\left(\tau_{j}, j\right)$ as an arc and $i$ as a fixed point, whereas $P_{\omega_{i, j}(\tau)}$ contains $\left(\tau_{j}, i\right)$ as an arc and $j$ as a fixed point. Thus, the relation $R_{s, t}\left(\omega_{i, j}(\tau)\right)>R_{s, t}(\tau)$ holds only if $\tau_{j}, i \in\{s, \ldots, t\}$ and $j \notin\{s, \ldots, t\}$, that is, only if $s \leq \tau_{j}<i=\tau_{i} \leq t<j$.

- If $(i, j)$ satisfies condition (b) of the definition of $I_{1}^{+}$, that is, $i<j=\tau_{j}<$ $\tau_{i}$, then similarly the fact that $R_{s, t}\left(\omega_{i, j}(\tau)\right)>R_{s, t}(\tau)$ implies that $j, \tau_{i} \in$ $\{s, \ldots, t\}$ and $i \notin\{s, \ldots, t\}$. Thereby, $i<s \leq j=\tau_{j}<\tau_{i} \leq t$ in this case.

- Assume that $(i, j)$ satisfies condition (c) of the definition of $I_{1}^{+}$, that is, $i<$ $j<\tau_{i}<\tau_{j}$. In this case, $\tau, \omega_{i, j}(\tau)$ differ by the fact that $\left(i, \tau_{i}\right),\left(j, \tau_{j}\right)$ are $\operatorname{arcs}$ of $P_{\tau}$, whereas $\left(i, \tau_{j}\right),\left(j, \tau_{i}\right)$ are $\operatorname{arcs}$ of $P_{\omega_{i, j}(\tau)}$. Then, we can see that we 
have $R_{s, t}\left(\omega_{i, j}(\tau)\right)>R_{s, t}(\tau)$ only for $j, \tau_{i} \in\{s, \ldots, t\}$ and $i, \tau_{j} \notin\{s, \ldots, t\}$. Whence, $i<s \leq j<\tau_{i} \leq t<\tau_{j}$.

- Next, assume that $(i, j)$ satisfies condition (d) of the definition of $I_{1}^{+}$, so $\tau_{j}<i<j<\tau_{i}$. Here, the only difference between $\tau$ and $\omega_{i, j}(\tau)$ is that $\left(i, \tau_{i}\right),\left(\tau_{j}, j\right)$ are $\operatorname{arcs}$ of $P_{\tau}$, whereas $\left(\tau_{j}, i\right),\left(j, \tau_{i}\right)$ are arcs of $P_{\omega_{i, j}(\tau)}$. Thus, the fact that $R_{s, t}\left(\omega_{i, j}(\tau)\right)>R_{s, t}(\tau)$ implies that we have either $\left(\tau_{j}, i \in\{s, \ldots, t\}\right.$ and $\left.j, \tau_{i} \notin\{s, \ldots, t\}\right)$ or $\left(j, \tau_{i} \in\{s, \ldots, t\}\right.$ and $\left.\tau_{j}, i \notin\{s, \ldots, t\}\right)$. Therefore, in this case, we have either $s \leq \tau_{j}<i \leq t<j<\tau_{i}$ or $\tau_{j}<i<s \leq j<\tau_{i} \leq t$.

Finally, this analysis reveals that only two situations can happen: either

(1) $s \leq \tau_{j}<i \leq t<j$ and $\tau_{i} \geq i$, or

(2) $i<s \leq j<\tau_{i} \leq t$ and $\left(\tau_{j}=j\right.$ or $\left.\tau_{j} \notin\{s, \ldots, t\}\right)$.

Now, let us consider successively the situations (1), (2). In both cases, we determine through formula $(*)$ a linear equation satisfied in the tangent space $\mathcal{T}$, that is, we construct an element of the space $\mathcal{T}^{\perp}$. To this end, we start with an arbitrary element $F^{\prime}=\left(V_{0}, \ldots, V_{n}\right) \in \Omega \cap \overline{\mathcal{Z}_{\sigma}}$ with $V_{l}=\left\langle f_{1}, \ldots, f_{l}\right\rangle_{\mathbb{K}}$ for all $l$, where $f_{l}=e_{l}+\sum_{m>l} \varphi_{l, m}\left(F^{\prime}\right) e_{m}$ as in Section 4.1. Recall that we have fixed indices $i_{1}<\ldots<i_{r}$ with $s \leq \tau_{i_{p}}<i_{p} \leq t$ for each $p$.

(1) We consider the family of vectors $\left(f_{1}, \ldots, f_{s-1}, x\left(f_{i_{1}}\right), \ldots, x\left(f_{i_{r}}\right), x\left(f_{i}\right)\right)$. This family comprises $s+r$ vectors that all lie in the subspace $V_{s-1}+x\left(V_{t}\right)$. In view of formula $(*)$, the family is linearly dependent. Thus, the matrix of the family in the basis $\left(e_{1}, \ldots, e_{n}\right)$ has rank $<s+r$. In particular, the minor determinant with respect to the sub-basis $\left(e_{1}, \ldots, e_{s-1}, e_{\tau_{i_{1}}}, \ldots, e_{\tau_{i_{r}}}, e_{\tau_{j}}\right)$ is equal to zero. The nullity of this minor determinant yields a polynomial relation satisfied on $\Omega \cap \overline{\mathcal{Z}_{\sigma}}$. Let us describe this relation more precisely. Note that $x\left(f_{i_{p}}\right)=e_{\tau_{i_{p}}}+$ $\sum_{l \in L_{i_{p}}} \varphi_{i_{p}, l}\left(F^{\prime}\right) e_{\tau_{l}}$ for each $p$, where $L_{i_{p}}:=\left\{l>i_{p}: \tau_{l}<l\right\}$. Furthermore, since $\tau_{i} \geq i$ and $\tau_{j}<j$, we have $x\left(e_{i}\right)=0, x\left(e_{j}\right)=e_{\tau_{j}}$ and thus $x\left(f_{i}\right)=$ $\varphi_{i, j}\left(F^{\prime}\right) e_{\tau_{j}}+\sum_{l \in L_{i} \backslash\{j\}} \varphi_{i, l}\left(F^{\prime}\right) e_{\tau_{l}}$ where $L_{i}:=\left\{l>i: \tau_{l}<l\right\}$. Therefore, a relation of the following form holds on $\Omega \cap \overline{\mathcal{Z}_{\sigma}}$ :

$$
\left|\begin{array}{ccccc}
1 & * & \cdots & (*) & * \\
* & 1 & & \vdots & * \\
\vdots & & \ddots & & \vdots \\
& (*) & & 1 & * \\
& & \ldots & * & \varphi_{i, j}
\end{array}\right|=0
$$

where the coefficients of the matrix marked with the symbol $*$ are either $\varphi_{l, m}$ for some $l<m$, or zero. Developing along the last column, this relation can be written $\varphi_{i, j}=P$, where $P$ is a polynomial in the $\varphi_{l, m}$ 's with no term of degree $\leq 1$. The differential at 0 of $\varphi_{i, j}-P$ therefore vanishes on the tangent space $\mathcal{T}$. This differential is simply $\varphi_{i, j}$, whence $\varphi_{i, j} \in \mathcal{T}^{\perp}$.

(2) Here, we consider the family of vectors

$$
\left(f_{1}, \ldots, f_{i-1}, f_{i+1}, \ldots, f_{s-1}, x\left(f_{i_{1}}\right), \ldots, x\left(f_{i_{r}}\right), f_{i}, x\left(f_{\tau_{i}}\right)\right) .
$$


As above, this family comprises $r+s$ vectors, all lying in $V_{s-1}+x\left(V_{t}\right)$, so that formula $(*)$ implies that the family is linearly dependent. Thus, the matrix of the family in the basis $\left(e_{1}, \ldots, e_{n}\right)$ has rank $<s+r$, hence the minor determinant with respect to the sub-basis $\left(e_{1}, \ldots, e_{i-1}, e_{i+1}, \ldots, e_{s-1}, e_{\tau_{i_{1}}}, \ldots, e_{\tau_{i_{r}}}\right.$, $\left.e_{i}, e_{j}\right)$ is equal to zero (here we use the assumption $\left(\tau_{j}=j\right.$ or $\tau_{j} \notin\{s, \ldots, t\}$ ), which guarantees that $j \notin\left\{\tau_{i_{1}}, \ldots, \tau_{i_{r}}\right\}$, so that the vectors in the tuple are all distinct and form indeed a sub-basis). The nullity of this minor determinant provides a polynomial relation on $\Omega \cap \overline{\mathcal{Z}_{\sigma}}$ that we describe now more carefully. As in situation (1), one has $x\left(f_{i_{p}}\right)=e_{\tau_{i_{p}}}+\sum_{l \in L_{i_{p}}} \varphi_{i_{p}, l}\left(F^{\prime}\right) e_{\tau_{l}}$ for each $p$. Since $i<\tau_{i}$, one has $x\left(e_{\tau_{i}}\right)=e_{i}$, thus $x\left(f_{\tau_{i}}\right)=e_{i}+\sum_{l \in L_{\tau_{i}}} \varphi_{\tau_{i}, l}\left(F^{\prime}\right) e_{\tau_{l}}$ with $L_{\tau_{i}}:=\left\{l>\tau_{i}: \tau_{l}<l\right\}$. Hence a relation of the following form is satisfied on $\Omega \cap \overline{\mathcal{Z}_{\sigma}}$ :

$$
\left|\begin{array}{ccccc}
1 & * & \cdots & (*) & * \\
* & \ddots & \ddots & \vdots & \vdots \\
\vdots & \ddots & 1 & * & * \\
(*) & \cdots & * & 1 & 1 \\
* & \cdots & * & \varphi_{i, j} & \psi
\end{array}\right|=0
$$

where as before the symbol $*$ is either $\varphi_{l, m}$ for some $l<m$, or zero, and where we have either $\psi=\varphi_{\tau_{i}, \tau_{j}}$ (if $\tau_{j} \in L_{\tau_{i}}$ ) or $\psi=0$ (otherwise). Developing the determinant along the last column, the relation becomes $\varphi_{i, j}-\psi=P$, where $P$ is a polynomial in the $\varphi_{l, m}$ 's with no term of degree $\leq 1$. Differentiating at 0 , we deduce that the relation $\varphi_{i, j}-\psi=0$ holds on the tangent space $\mathcal{T}$. Therefore, $\varphi_{i, j}-\psi \in \mathcal{T}^{\perp}$. This completes the proof of Proposition 2(iii).

4.6. Proof of Proposition 2(iv). We first note that the set $I_{2}$ is formed by the pairs $(i, j), 1 \leq i<j \leq n$, which satisfy at least one of the following conditions:

(a) $i \leq \tau_{j}<j$ and $i \leq \tau_{i}$;

(b) $i<\tau_{i}<j$.

Indeed, these are the only configurations that are not represented in $I_{0}, I_{1}^{-}, I_{1}^{+}$.

Take an arbitrary element $F^{\prime}=\left(\left\langle f_{1}, \ldots, f_{l}\right\rangle_{\mathbb{K}}\right)_{l=0}^{n} \in \Omega \cap \overline{\mathcal{Z}_{\sigma}}$, where $f_{l}=e_{l}+$ $\sum_{m>l} \varphi_{l, m}\left(F^{\prime}\right) e_{m}$ as before. We will exploit the property of $F^{\prime}$ to be $x$-stable.

First, assume that $(i, j)$ satisfies condition (a) above. In this case, one has $x\left(e_{i}\right)=0$ and $x\left(e_{j}\right)=e_{\tau_{j}}$, so that $x\left(f_{i}\right)=\varphi_{i, j}\left(F^{\prime}\right) e_{\tau_{j}}+\sum_{l \in L_{i} \backslash\{j\}} \varphi_{i, l}\left(F^{\prime}\right) e_{\tau_{l}}$ where $L_{i}=$ $\left\{l>i: \tau_{l}<l\right\}$. Since $F^{\prime}$ is $x$-stable, the family of vectors $\left(f_{1}, \ldots, f_{i-1}, x\left(f_{i}\right)\right)$ is linearly dependent. Thus, the matrix of $\left(f_{1}, \ldots, f_{i-1}, x\left(f_{i}\right)\right)$ with respect to the basis $\left(e_{1}, \ldots, e_{n}\right)$ has rank $<i$. In particular, the minor determinant with respect to the sub-basis $\left(e_{1}, \ldots, e_{i-1}, e_{\tau_{j}}\right)$ is zero. The nullity of this minor determinant yields a polynomial relation that is satisfied on the subset $\Omega \cap \overline{\mathcal{Z}_{\sigma}}$. This relation takes the form

$$
\left|\begin{array}{ccccc}
1 & 0 & \cdots & (0) & * \\
* & 1 & & \vdots & * \\
\vdots & & \ddots & & \vdots \\
& (*) & & 1 & * \\
& & \ldots & * & \varphi_{i, j}
\end{array}\right|=0
$$


where the coefficients of the matrix represented with the star $*$ are either $\varphi_{l, m}$ for some $l, m$, or zero. Developing along the last column, the relation becomes $\varphi_{i, j}=P$, where $P$ is a polynomial in the $\varphi_{l, m}$ 's with no term of degree $\leq 1$. The differential at 0 of $\varphi_{i, j}-P$ is therefore a linear form which vanishes on the tangent space $\mathcal{T}$, whence $\varphi_{i, j} \in \mathcal{T}^{\perp}$.

Next, assume that $(i, j)$ satisfies condition (b). Hence, $x\left(e_{\tau_{i}}\right)=e_{i}$, so that $x\left(f_{\tau_{i}}\right)=$ $e_{i}+\sum_{l \in L_{\tau_{i}}} \varphi_{\tau_{i}, l}\left(F^{\prime}\right) e_{\tau_{l}}$, where $L_{\tau_{i}}=\left\{l>\tau_{i}: \tau_{l}<l\right\}$. Since $F^{\prime}$ is $x$-stable, we see that the family of vectors $\left(f_{1}, \ldots, f_{i-1}, f_{i+1}, \ldots, f_{\tau_{i}-1}, f_{i}, x\left(f_{\tau_{i}}\right)\right)$ is linearly dependent. Thus, its matrix in the basis $\left(e_{1}, \ldots, e_{n}\right)$ has rank $<\tau_{i}$, and so the minor determinant with respect to the sub-basis $\left(e_{1}, \ldots, e_{i-1}, e_{i+1}, \ldots, e_{\tau_{i}-1}, e_{i}, e_{j}\right)$ is zero. This yields a polynomial relation of the following form satisfied on the subset $\Omega \cap \overline{\mathcal{Z}_{\sigma}}$ :

$$
\left|\begin{array}{ccccc}
1 & & (0) & * & * \\
* & \ddots & & \vdots & * \\
\vdots & \ddots & 1 & * & \vdots \\
(*) & \cdots & * & 1 & 1 \\
* & \cdots & * & \varphi_{i, j} & \psi
\end{array}\right|=0
$$

where, as above, each star $*$ is either 0 or $\varphi_{l, m}$ for some $l, m$, and where either $\psi=\varphi_{\tau_{i}, \tau_{j}}$ (if $\tau_{j} \in L_{\tau_{i}}$ ) or $\psi=0$ (otherwise). Developing the determinant along the last column, we get $\varphi_{i, j}-\psi=P$, where $P$ is a polynomial in the $\varphi_{l, m}$ 's with no term of degree $\leq 1$. Differentiating at 0 , we deduce that the relation $\varphi_{i, j}-\psi=0$ is satisfied on the tangent space $\mathcal{T}$, whence $\varphi_{i, j}-\psi \in \mathcal{T}^{\perp}$. This achieves the proof of Proposition 2(iv).

\section{References}

[1] R. Bezrukavnikov, I. Mirković and D. Rumynin, Localization of modules for a semisimple Lie algebra in prime characteristic, Ann. Math. (2) 167(3) (2008), 945-991. With an appendix by R. Bezrukavnikov and S. Riche.

[2] L. Fresse, Singular components of Springer fibers in the two-column case, Ann. Inst. Fourier (Grenoble) 59(6) (2009), 2429-2444.

[3] L. Fresse and A. Melnikov, On the singularity of the irreducible components of a Springer fiber in $\operatorname{sl}(n)$, Selecta Math. (N.S.) 16(3) (2010), 393-418.

[4] - Some characterizations of singular components of Springer fibers in the two-column case, Algebr. Represent. Theory 14(6) (2011), 1063-1086.

[5] F.Y.C. Fung, On the topology of components of some Springer fibers and their relation to Kazhdan-Lusztig theory, Adv. Math. 178(2) (2003), 244-276.

[6] D. Kazhdan and G. Lusztig, Proof of the Deligne-Langlands conjecture for Hecke algebras, Invent. Math. 87(1) (1987), 153-215.

[7] V. Lakshmibai and C.S. Seshadri, Singular locus of a Schubert variety, Bull. Amer. Math. Soc. (N.S.) 11(2) (1984), 363-366.

[8] A. Melnikov, B-orbits of nilpotent order 2 and link patterns, Preprint (2007). arXiv:0703371

[9] N. Perrin and E. Smirnov, Springer fiber components in the two columns case for types $A$ and $D$ are normal, Bull. Soc. Math. France, to appear.

[10] N. Spaltenstein, On the fixed point set of a unipotent element on the variety of Borel subgroups, Topology 16(2) (1977), 203-204.

[11] - Classes unipotentes et sous-groupes de Borel, Lecture Notes in Mathematics, 946, Springer-Verlag, Berlin (1982), ISBN 3-540-11585-4. 
[12] T.A. Springer, Trigonometric sums, Green functions of finite groups and representations of Weyl groups, Invent. Math. 36 (1976), 173-207.

[13] - A construction of representations of Weyl groups, Invent. Math. 44(3) (1978), 279-293.

Département de Mathématiques, Université de Cergy-Pontoise, 95300 Cergy-Pontoise, FRANCE

E-mail address: Lucas.Fresse@u-cergy.fr 